\title{
On the Feynman path integral for the Dirac equation in the general dimensional spacetime
}

\author{
Wataru Ichinose* \\ Department of Mathematical Sciences, Shinshu University, Matsumoto \\ 390-8621, Japan. \\ E-mail: ichinose@math.shinshu-u.ac.jp
}

\begin{abstract}
The Feynman path integral for the Dirac equation in the general dimensional spacetime is determined mathematically in the form of the sum-over-histories satisfying the superposition principle, i.e. the "sum" of the probability amplitudes with a common weight, over all possible paths that go in any direction at any speed forward and backward in time. It should be noted that our Feynman path integral is determined not in configuration space, but in phase space.
\end{abstract}

\section{Introduction}

Let $T>0$ be an arbitrary constant, $0 \leq t \leq T$ and $x=\left(x_{1}, \ldots, x_{d}\right) \in$ $R^{d}$. Let $E(t, x)=\left(E_{1}, \ldots, E_{d}\right) \in R^{d}$ and $\left(B_{j k}(t, x)\right)_{1 \leq j<k \leq d} \in R^{d(d-1) / 2}$ de-

${ }^{*}$ Research partially supported by Grant-in-Aid for Scientific Research No.23540195, Ministry of Education, Culture, Sports, Science and Technology, Japanese Government. 
note the electric strength and the magnetic strength tensor, respectively and $(V(t, x), A(t, x))=\left(V, A_{1}, \ldots, A_{d}\right) \in R^{d+1}$ an electromagnetic potential, i.e.

$$
\begin{aligned}
& E=-\frac{\partial A}{\partial t}-\frac{\partial V}{\partial x} \\
& B_{j k}=\frac{\partial A_{k}}{\partial x_{j}}-\frac{\partial A_{j}}{\partial x_{k}} \quad(1 \leq j<k \leq d),
\end{aligned}
$$

where $\partial V / \partial x=\left(\partial V / \partial x_{1}, \ldots, \partial V / \partial x_{d}\right)$. We consider the Dirac equation

$$
\begin{aligned}
& i \hbar \frac{\partial u}{\partial t}(t)=H(t) u(t) \\
& :=\left[c \sum_{j=1}^{d} \widehat{\alpha}^{(j)}\left(\frac{\hbar}{i} \frac{\partial}{\partial x_{j}}-e A_{j}(t, x)\right)+\widehat{\beta} m c^{2}+e V(t, x)\right] u(t)
\end{aligned}
$$

as in (11) of $\S 67$, p.257 of [4] or (4.19), p.70 of [11], where ${ }^{t} u(t)=\left(u_{1}(t), \ldots, u_{N}(t)\right) \in$ $\mathbb{C}^{N}, \widehat{\alpha}^{(j)}(j=1,2, \ldots, d)$ and $\widehat{\beta}$ are constant $N \times N$ Hermitian matrices, $c$ is the velocity of light, $\hbar$ is the Plank constant and $e$ is the charge of an electron. For the sake of simplicity we suppose $\hbar=1$ and $e=1$ hereafter. We note that through the present paper constant matrices $\widehat{\alpha}^{(j)}(j=1,2, \ldots, d)$ and $\widehat{\beta}$ are assumed to be only Hermitian.

Let us take the Hamiltonian function

$$
\mathcal{H}(t, x, p)=c \sum_{j=1}^{d} \widehat{\alpha}^{(j)}\left(p_{j}-A_{j}(t, x)\right)+\widehat{\beta} m c^{2}+V(t, x)
$$

as in (23) of $\S 69$, p.261 of [4], where $p \in R^{d}$ is the canonical momentum. It follows from the classical rule of the replacement as in $\S 67$, p.257 of [4] that $\xi=p-A(t, x) \in R^{d}$ is the kinetic momentum. So, the Lagrangian function is given by

$$
\begin{aligned}
& \mathcal{L}(t, x, \xi)=p \cdot \dot{x}-\mathcal{H}(t, x, p) \\
& =\xi \cdot \dot{x}+\dot{x} \cdot A(t, x)-V(t, x)-\left(c \widehat{\alpha} \cdot \xi+\widehat{\beta} m c^{2}\right),
\end{aligned}
$$


where $p \cdot \dot{x}=\sum_{j=1}^{d} p_{j} \dot{x}_{j}, \widehat{\alpha}=\left(\widehat{\alpha}^{(1)}, \ldots, \widehat{\alpha}^{(d)}\right)$ and $\widehat{\alpha} \cdot \xi=\sum_{j=1}^{d} \widehat{\alpha}^{(j)} \xi_{j}$.

We will determine the Feynman path integral in phase space in terms of (1.4). Let $0 \leq \tau_{j} \leq T(j=1,2, \ldots, \nu-1)$ and set $\Delta:=\left\{\tau_{j}\right\}_{j=1}^{\nu-1}$. We don't necessarily assume $\tau_{j} \leq \tau_{j+1}$. We set $\tau_{0}=0$ and $\tau_{\nu}=t$. Let $x \in R^{d}$ be fixed. For arbitrary points $x^{(j)} \in R^{d}(j=0,1, \ldots, \nu-1)$ and $\xi^{(j)} \in$ $R^{d}(j=0,1, \ldots, \nu-1)$ let us denote the broken line path in $R^{d}$ joining $x^{(j)}$ at $\tau_{j}\left(j=0,1, \ldots, \nu, x^{(\nu)}=x\right)$ in order by $q_{\Delta}\left(x^{(0)}, \ldots, x^{(\nu-1)}, x\right)$ and the piecewise constant path in $R^{d}$ taking value $\xi^{(j)}(j=0,1, \ldots, \nu-1)$ for $\theta \in\left[\tau_{j}, \tau_{j+1}\right)$ if $\tau_{j}<\tau_{j+1}$ or $\theta \in\left(\tau_{j+1}, \tau_{j}\right]$ if $\tau_{j+1}<\tau_{j}$ and $\xi^{(\nu-1)}$ at $t$ by $\xi_{\Delta}\left(\xi^{(0)}, \ldots, \xi^{(\nu-1)}\right)$. We note that the paths $q_{\Delta}$ and $\xi_{\Delta}$ go in any direction forward and backward in time and that $q_{\Delta}$ have any speed. Let us write $q_{\Delta}$ and $\xi_{\Delta}$ for $\theta \in\left[\tau_{j}, \tau_{j+1}\right)$ or $\left(\tau_{j+1}, \tau_{j}\right]$ by $q_{\Delta}(\theta)$ and $\xi_{\Delta}(\theta)$.

The approximation $K_{D \Delta}(t, 0) f$ of the Feynman path integral for the Dirac equation (1.2) is determined in terms of the oscillatory integral (cf. p.45 of [23]) by

$$
\begin{aligned}
& K_{D \Delta}(t, 0) f=\iint e^{* i S\left(t, q_{\Delta}, \xi_{\Delta}\right)} f\left(q_{\Delta}(0)\right) \mathcal{D} q_{\Delta} \mathcal{D} \xi_{\Delta} \\
& :=\mathrm{Os}-\int \cdots \int e^{* i S\left(t, q_{\Delta}, \xi_{\Delta}\right)} f\left(x^{(0)}\right) d x^{(0)} \cdots d x^{(\nu-1)} d \xi^{(0)} \cdots d \xi^{(\nu-1)}
\end{aligned}
$$

for $f \in \mathcal{S}\left(R^{d}\right)^{N}$, i.e. the Schwartz rapidly decreasing function, where $d \xi^{(j)}=$ $(2 \pi)^{-d} d \xi^{(j)}$ and the probability amplitude $\exp * i S\left(t, q_{\Delta}, \xi_{\Delta}\right)$ for a path $\left(q_{\Delta}, \xi_{\Delta}\right)$ is defined as a product of matirices from the Lagrangian function (1.4) by

$$
\begin{aligned}
& \exp i \sum_{j=0}^{\nu-1} \int_{\tau_{j}}^{\tau_{j+1}}\left\{\xi_{\Delta}(\theta) \cdot \dot{q}_{\Delta}(\theta)+\dot{q}_{\Delta}(\theta) \cdot A\left(\theta, q_{\Delta}(\theta)\right)-V\left(\theta, q_{\Delta}(\theta)\right)\right\} d \theta \\
& \times \exp -i \int_{\tau_{\nu-1}}^{t}\left\{c \widehat{\alpha} \cdot \xi_{\Delta}(\theta)+\widehat{\beta} m c^{2}\right\} d \theta \cdot \exp -i \int_{\tau_{\nu-2}}^{\tau_{\nu-1}}\left\{c \widehat{\alpha} \cdot \xi_{\Delta}(\theta)+\widehat{\beta} m c^{2}\right\} d \theta \\
& \cdots \exp -i \int_{0}^{\tau_{1}}\left\{c \widehat{\alpha} \cdot \xi_{\Delta}(\theta)+\widehat{\beta} m c^{2}\right\} d \theta
\end{aligned}
$$


A little simpler definition of $\exp * i S\left(t, q_{\Delta}, \xi_{\Delta}\right)$ than (1.6) will be given in Remark 2.3 of the present paper.

The main results of the present paper are stated in Theorems 2.1 and 2.2. Let $L^{2}\left(R^{d}\right)$ denote the space of all square integrable functions in $R^{d}$ with inner product $(f, g):=\int f(x) \overline{g(x)} d x$ and norm $\|f\|$, where $\overline{g(x)}$ denotes the complex conjugate of $g(x)$. Let $f \in L^{2}\left(R^{d}\right)^{N}$ and $L_{0} \geq 0$ an arbitrary constant. Then, we will prove in Theorem 2.1 that as $|\Delta|:=\max _{0 \leq j \leq \nu-1}\left|\tau_{j+1}-\tau_{j}\right| \rightarrow 0$ under the assumption

$$
\sum_{j=0}^{\nu-1}\left|\tau_{j+1}-\tau_{j}\right| \leq L_{0},
$$

then $K_{D \Delta}(t, 0) f$ converges to $K_{D}(t, 0) f$ independently of the choice of $L_{0}$, which we call the Feynman path integral, in $L^{2}\left(R^{d}\right)^{N}$ uniformly in $t \in[0, T]$ and that $K_{D}(t, 0) f$ is the solution to $(1.2)$ with $u(0)=f$. In Theorem 2.2 we will prove the convergence of $K_{D}(t, 0) f$ to $K_{D}(t, 0) f$ in some weighted Soblolev spaces as $|\Delta| \rightarrow 0$ uniformly in $t \in[0, T]$ under the assumption (1.7).

We could say from (1.5) that the Feynman path integral $K_{D}(t, 0) f$ is written in the form of the "sum" of the probability amplitudes with a common weight over all possible paths, i.e. the form of the sum-over-histories satisfying the superposition principle as is stated in sections 1-4, 2-2, 2-5 and 2-6 of [9], in (13) and (14), p.752 of [6], in (2) and (4), p.772 of [7], in (37) and (38), p.447 of [8], in p.146 of [22], in (9.2), p.276 of [25] and in $§ 2.3$ of [27]. As Feynman noted in p.376 of [5] that the electron goes in any direction at any speed forward or backward in time, we consider all possible paths to determine $K_{D}(t, 0) f$ along which the electron goes in any direction at any speed foward or backward in time. We remark that Feynman also noted for the probability amplitude of a photon in pp.88 and 89 of [10] that there is also an amplitude of a photon, i.e. light to go faster (or slower) than the conventional speed $c$ of 
light and not to go only in straight lines.

We note that it is stated in $§ 2-6$, p.38, $\S 9-1$, p.237 and $\S 9-8$, p.264 of [9] that in the relativistic theory of the electron we shall not find it possible to express the amplitude for a path as $e^{i S}$, or in any other simple way.

It should be also noted that the paths $q_{\Delta}$ and $\xi_{\Delta}$ to determine $K_{D \Delta}(t, 0) f$ were also used to determine the Feynman path integrals for the Schrödinger equation in Main Theorem of [18] and the Pauli equation in Theorem 2.3 of [20], though we didn't consider the paths that go backward in time, but could consider as is seen from their proofs.

Now we go back to the past studies of the Feynman path integral for the Dirac equation. There seems to be no past studies of the Feynman path integral in phase space. All studies seem to be made of that in configuration space. Consider the Dirac equation in two dimensional spacetime

$$
i \frac{\partial u}{\partial t}(t)=\left[c \widehat{\alpha}^{(0)}\left(\frac{1}{i} \frac{\partial}{\partial x}-A(t, x)\right) u+\widehat{\beta}^{(0)} m c^{2}+V(t, x)\right] u(t),
$$

where ${ }^{t} u(t)=\left(u_{1}(t), u_{2}(t)\right) \in \mathbb{C}^{2}$ and

$$
\widehat{\alpha}^{(0)}=\left(\begin{array}{cc}
1 & 0 \\
0 & -1
\end{array}\right), \quad \widehat{\beta}^{(0)}=\left(\begin{array}{cc}
0 & -1 \\
-1 & 0
\end{array}\right) .
$$

Let $(V, A)=0$ in (1.8). Suppose that the interval $[0, t]$ is divided into small equal steps of length $\epsilon_{0}>0$. We consider zigzags in the spacetime of straight segments with velocity $c$ that go only forward in time. The amplitude for each zigzag is given by $\left(i \epsilon_{0}\right)^{R}$, where $R$ is the number of reversals. It follows from the superposition principle that the Feynman path integral was determined by (2-27), p.35 of [9]. See Appendix E, p.118 of [26] in detail. The other studies below seem not to suppose the superposition principle and also consider the paths that go backward in time. In [13] and Theorem 2.1 of [14] the solution to 
a general (1.8) was written in terms of a measure on the space of all continuous paths in $[0, t]$. In Theorem, p.8 of [1] and p.221 of [3] the solution to (1.8) was written in terms of a Poisson process. It is noted that in $[1,3,13,14]$ the support of the measure on the path space is included in the space consisting of all zigzags with velocity $c$ that go only forward in time.

In Theorem, p.318 of [12] the Fourier transform $\hat{u}(t, \xi)=\int e^{-i x \cdot \xi} u(t, x) d x$ of the solution $u(t, x)$ to $(1.2)$ is represented in terms of a Poisson measure, where $A_{j}(x)(j=0,1, \ldots, d)\left(A_{0}=V / c\right)$ are assumed to be independent of $t$ and given by the Fourier transform of totally finite measures. In (5), p.2436 and (35), p.2448 of [21] the physical consideration is given to the representation of the solution to (1.2) with $d=3$ and $N=4$ that generalizes the result to (1.8) in [9] stated above.

We will explain an idea for proving our results. Let $0 \leq s \leq t \leq T$ or $0 \leq t \leq s \leq T$. We define

$$
q_{x, y}^{t, s}(\theta)=y+\frac{\theta-s}{t-s}(x-y)
$$

in $s \leq \theta \leq t$ or $t \leq \theta \leq s$ for $x$ and $y$ in $R^{d}$ and consider the classical action in phase space

$$
\begin{aligned}
& S(t, s ; x, \xi, y):=\int_{s}^{t}\left\{\xi \cdot \dot{q}_{x, y}^{t, s}(\theta)+\dot{q}_{x, y}^{t, s}(\theta) \cdot A\left(\theta, q_{x, y}^{t, s}(\theta)\right)\right. \\
& \left.\quad-V\left(\theta, q_{x, y}^{t, s}(\theta)\right)-\left(c \widehat{\alpha} \cdot \xi+\widehat{\beta} m c^{2}\right)\right\} d \theta \\
& =(x-y) \cdot \xi+\int_{s}^{t}\left\{\dot{q}_{x, y}^{t, s}(\theta) \cdot A\left(\theta, q_{x, y}^{t, s}(\theta)\right)-V\left(\theta, q_{x, y}^{t, s}(\theta)\right)\right\} \\
& \quad-(t-s)\left(c \widehat{\alpha} \cdot \xi+\widehat{\beta} m c^{2}\right)
\end{aligned}
$$

from (1.4), where $\dot{q}_{x, y}^{t, s}(\theta)=d q_{x, y}^{t, s}(\theta) / d \theta$. We take $\chi \in C_{0}^{\infty}\left(R^{d}\right)$, i.e. an infinitely differentiable function in $R^{d}$ with compact support, such that $\chi(0)=1$. Let 
$\epsilon>0$ be a constant and define an operator

$$
\left(G_{\epsilon}(t, s) f\right)(x)=\iint e^{i S(t, s ; x, \xi, y)} f(y) \chi(\epsilon \xi) d y d \xi
$$

for $\mathcal{S}\left(R^{d}\right)^{N}$. Then we can write

$$
K_{D \Delta}(t, 0) f=\lim _{\epsilon \rightarrow 0} G_{\epsilon}\left(t, \tau_{\nu-1}\right) \chi(\epsilon \cdot) G_{\epsilon}\left(\tau_{\nu-1}, \tau_{\nu-2}\right) \chi(\epsilon \cdot) \cdots \chi(\epsilon \cdot) G_{\epsilon}\left(\tau_{1}, 0\right) f
$$

from (1.5) and (1.6). We can prove the main results from (1.12) as in the proof of Theorem of [16], which is originated in [24].

In more detail we will prove that $\left\{G_{\epsilon}(t, s)\right\}_{0<\epsilon \leq 1}$ is a bounded family of operators from $\mathcal{S}\left(R^{d}\right)^{N}$ into itself, that there exists an operator $G(t, s)$ on $\mathcal{S}\left(R^{d}\right)^{N}$ independent of the choice of $\chi$ satisfying

$$
G(t, s) f=\lim _{\epsilon \rightarrow 0} G_{\epsilon}(t, s) f
$$

in $\mathcal{S}\left(R^{d}\right)^{N}$ for $f \in \mathcal{S}\left(R^{d}\right)^{N}$, that the stability

$$
\|G(t, s) f\| \leq e^{K(t-s)^{2}}\|f\|
$$

holds with a constant $K \geq 0$ and that the consistency

$$
\lim _{\epsilon \rightarrow 0}\left\|\left(i \frac{\partial}{\partial t}-H(t)\right) G_{\epsilon}(t, s) f\right\| \leq C(t-s)\left\|<\cdot>^{M} f\right\|
$$

holds with a constant $C \geq 0$ and a positive integer $M$, where $\langle x\rangle=$ $\sqrt{1+|x|^{2}}$ and $\|f\|^{2}=\sum_{j=1}^{N}\left\|f_{j}\right\|^{2}$ for ${ }^{t} f=\left(f_{1}, \ldots, f_{N}\right) \in L^{2}\left(R^{d}\right)^{N}$.

Let $U(t, 0) f$ for $f \in \mathcal{S}\left(R^{d}\right)^{N}$ be the solution to (1.2) with $u(0)=f$. From (1.12) and (1.13) we can easily see

$$
K_{D \Delta}(t, 0) f=G\left(t, \tau_{\nu-1}\right) G\left(\tau_{\nu-1}, \tau_{\nu-2}\right) \cdots G\left(\tau_{1}, 0\right) f
$$

for $f \in \mathcal{S}\left(R^{d}\right)^{N}$. From (1.14) and (1.15) we will prove that

$$
\left\|K_{D \Delta}(t, 0) f-U(t, 0) f\right\|=\left\|G\left(t, \tau_{\nu-1}\right) \cdots G\left(\tau_{1}, 0\right) f-U\left(t, \tau_{\nu-1}\right) \cdots U\left(\tau_{1}, 0\right) f\right\|
$$


for $f \in L^{2}\left(R^{d}\right)^{N}$ converges to 0 uniformly in $t \in[0, T]$ as $|\Delta| \rightarrow 0$ under the assumption (1.7). The convergence of $K_{D \Delta}(t, 0) f$ in the weighted Sobolev spaces as $|\Delta| \rightarrow 0$ under (1.7) can be proved in the same way. We note that the theory of pseudo-differential operators plays an important role to prove (1.14), (1.15) and their generalizations.

The plan of the present paper is as follows. In $\S 2$ the main results in the present paper, i.e. Theorems 2.1 and 2.2 are stated. In sections 3 and 4 the stability and the consistency of $G(t, s)$ are proved, respectively. In $\S 5$ we give a complete proof of Theorems 2.1 and 2.2.

\section{Main results}

For an $x=\left(x_{1}, \ldots, x_{d}\right) \in R^{d}$ and a multi-index $\alpha=\left(\alpha_{1}, \ldots, \alpha_{d}\right)$ we write $|\alpha|=\sum_{j=1}^{d} \alpha_{j}, x^{\alpha}=x_{1}^{\alpha_{1}} \cdots x_{d}^{\alpha_{d}}, \partial_{x_{j}}=\partial / \partial x_{j}$ and $\partial_{x}^{\alpha}=\partial_{x_{1}}^{\alpha_{1}} \cdots \partial_{x_{d}}^{\alpha_{d}}$. Let $M$ and $a$ be positive integers. We introduce the weighted Sobolev spaces $B_{M}^{a}\left(R^{d}\right)^{N}:=$ $\left\{f \in L^{2}\left(R^{d}\right)^{N} ;\|f\|_{B_{M}^{a}}:=\|f\|+\sum_{|\alpha|=a M}\left\|x^{\alpha} f\right\|+\sum_{|\alpha|=a}\left\|\partial_{x}^{\alpha} f\right\|<\infty\right\}$. Let $B_{M}^{-a}\left(R^{d}\right)^{N}$ denote their dual spaces. We set $B_{M}^{0}\left(R^{d}\right)^{N}:=L^{2}\left(R^{d}\right)^{N}$.

Throughout the present paper we always assume for electromagnetic potentials $(V, A)$ that $V, \partial_{x_{j}} V, \partial_{t} A_{k}$ and $\partial_{x_{j}} A_{k}(j, k=1,2, \ldots, d)$ are continuous in $[0, T] \times R^{d}$. In the present paper we often use symbols $C, C_{\alpha}, C_{\alpha, \beta}$ and $C_{a}$ to write down constants, though these values are different in general. We note again that throughout the present paper constant matrices $\widehat{\alpha}^{(j)}(j=1,2, \ldots, d)$ and $\widehat{\beta}$ in (1.2) are assumed to be only Hermitian. The following are the main theorems in the present paper.

TheOREM 2.1. Suppose that $\partial_{x}^{\alpha} E_{j}(t, x)(j=1,2, \ldots, d), \partial_{x}^{\alpha} B_{j k}(t, x)(1 \leq$ 
$j<k \leq d)$ and $\partial_{t} B_{j k}(t, x)$ are continuous in $[0, T] \times R^{d}$ for all $\alpha$. We assume

$$
\begin{gathered}
\left|\partial_{x}^{\alpha} E_{j}(t, x)\right| \leq C_{\alpha}, \quad|\alpha| \geq 1 \\
\left|\partial_{x}^{\alpha} B_{j k}(t, x)\right| \leq C_{\alpha}<x>^{-\left(1+\delta_{\alpha}\right)}, \quad|\alpha| \geq 1
\end{gathered}
$$

in $[0, T] \times R^{d}$ with constants $\delta_{\alpha}>0$ for $j, k=1,2, \ldots, d$. We define $K_{D \Delta}(t, 0) f$ for $f \in \mathcal{S}^{N}$ by (1.5). Then we have: (1) $K_{D \Delta}(t, 0)$ on $\mathcal{S}^{N}$ can be extended to a bounded operator on $\left(L^{2}\right)^{N}$. (2) Let $f \in\left(L^{2}\right)^{N}$ and $L_{0} \geq 0$ be an arbitrary constant. Then, as $|\Delta| \rightarrow 0$ under the assumption (1.7), the approximation $K_{D \Delta}(t, 0) f$ converges to the Feynman path integral $K_{D}(t, 0) f$ in $\left(L^{2}\right)^{N}$ independent of the choice of $L_{0}$ uniformly in $t \in[0, T]$. (3) $K_{D}(t, 0) f$ for $f \in\left(L^{2}\right)^{N}$ belongs to $\mathcal{E}_{t}^{0}\left([0, T] ;\left(L^{2}\right)^{N}\right)$ and is the solution to the Dirac equation (1.2) in distribution sense with $u(0)=f$, where $\mathcal{E}_{t}^{j}\left([0, T] ;\left(L^{2}\right)^{N}\right)(j=0,1, \ldots)$ denotes the space of all $\left(L^{2}\right)^{N}$-valued j-times continuously differentiable functions in $t \in[0, T]$. (4) Let $0<t_{1}<t$. Then we have the rule for two events:

$$
K_{D}(t, 0) f=K_{D}\left(t, t_{1}\right) K_{D}\left(t_{1}, 0\right) f, K_{D}\left(t, t_{1}\right) f=K_{D}(t, 0) K_{D}\left(0, t_{1}\right) f .
$$

(5) Let $\psi(t, x)$ be a real-valued function such that $\partial_{x_{j}} \partial_{x_{k}} \psi(t, x)$ and $\partial_{t} \partial_{x_{j}} \psi(t, x)$ $(j, k=1,2, \ldots, d)$ are continuous in $[0, T] \times R^{d}$ and consider the gauge transformation

$$
V^{\prime}=V-\frac{\partial \psi}{\partial t}, \quad A_{j}^{\prime}=A_{j}+\frac{\partial \psi}{\partial x_{j}} .
$$

We write $(1.5)$ as $K_{D \Delta}^{\prime}(t, 0) f$ for this $\left(V^{\prime}, A^{\prime}\right)$. Then we have the formula

$$
K_{D \Delta}^{\prime}(t, 0) f=e^{i \psi(t, \cdot)} K_{D \Delta}(t, 0)\left(e^{-i \psi(0, \cdot)} f\right)
$$

for all $f \in\left(L^{2}\right)^{N}$.

Remark 2.1. The rule for two events (2.3) in Theorem 2.1 is stated by (2-31), p.37 of [9]. 
Example 2.1. Let us state an example of the family of $\Delta$ satisfying (1.7). Let $N \geq 1$ be an arbitrary integer. We consider the family of $\Delta=\left\{\tau_{j}\right\}_{j=1}^{\nu-1}$ :

$$
\begin{aligned}
& 0=\tau_{0}<\tau_{1}<\ldots<\tau_{l_{1}}>\tau_{l_{1}+1}>\ldots>\tau_{l_{1}^{\prime}}<\tau_{l_{1}^{\prime}+1}<\ldots<\tau_{l_{2}} \\
& >\tau_{l_{2}+1}>\ldots>\tau_{l_{2}^{\prime}}<\ldots<\tau_{l_{N}}>\ldots>\tau_{l_{N}^{\prime}}<\ldots<\tau_{\nu}=t .
\end{aligned}
$$

Then we have $\sum_{j=1}^{\nu}\left|\tau_{j}-\tau_{j-1}\right| \leq(2 N+1) T$. So (1.7) always holds.

TheOrem 2.2. We assume (2.1) and (2.2). In addition, we suppose

$$
\left|\partial_{x}^{\alpha} A_{j}(t, x)\right| \leq C_{\alpha}, \quad|\alpha| \geq 1
$$

in $[0, T] \times R^{d}$ for $j=1,2, \ldots, d$ and that there exists an integer $M \geq 1$ satisfying

$$
\left|\partial_{x}^{\alpha} V(t, x)\right| \leq C_{\alpha}<x>^{M}, \quad|\alpha| \geq 1
$$

and

$$
\left|\partial_{x}^{\alpha} \partial_{t} A_{j}(t, x)\right| \leq C_{\alpha}<x>^{M}
$$

for all $\alpha$ in $[0, T] \times R^{d}$. Then we have: (1) $K_{D \Delta}(t, 0)$ on $\mathcal{S}^{N}$ can be extended to a bounded operator on $\left(B_{M}^{a}\right)^{N}(a=0,1, \ldots)$. (2) Let $f \in\left(B_{M+1}^{a}\right)^{N}$ and $L_{0} \geq 0$ an arbitrary constant. Then, as $|\Delta| \rightarrow 0$ under the assumption (1.7), $K_{D \Delta}(t, 0) f$ converges to $K_{D}(t, 0) f$ in $\left(B_{M+1}^{a}\right)^{N}$ uniformly in $t \in[0, T]$.

Remark 2.2. We can easily see from (1.1) that under the assumptions of Theorem $2.2 \partial_{x}^{\alpha} E_{j}(t, x), \partial_{x}^{\alpha} B_{j k}(t, x)$ and $\partial_{t} B_{j k}(t, x)$ are continuous in $[0, T] \times R^{d}$ for all $\alpha$ and so that the assumptions of Theorem 2.1 hold.

Remark 2.3. Let $q_{\Delta}=q_{\Delta}\left(x^{(0)}, \ldots, x^{(\nu-1)}, x\right)$ and $\xi_{\Delta}=\xi_{\Delta}\left(\xi^{(0)}, \ldots, \xi^{(\nu-1)}\right)$ be the broken line path and the piecewise constant path respectively defined in $\S 1$. Let $\mathcal{L}(t, x, \xi)$ be the Lagrangian function defined by (1.4). Let us define 
$\mathcal{F}\left(\theta, \tau_{j} ; q_{\Delta}, \xi_{\Delta}\right)$ for $\theta \in\left[\tau_{j}, \tau_{j+1}\right)$ or $\theta \in\left(\tau_{j+1}, \tau_{j}\right](j=0,1, \ldots, \nu-1)$ by the solution to

$$
\frac{d}{d \theta} \mathcal{F}\left(\theta, \tau_{j}\right)=i \mathcal{L}\left(\theta, q_{\Delta}(\theta), \xi_{\Delta}(\theta)\right) \mathcal{F}\left(\theta, \tau_{j}\right)
$$

with $\mathcal{F}\left(\tau_{j}, \tau_{j}\right)=I_{N}$, where $I_{N}$ is an identity $N \times N$ matrix. Then we get

$$
\exp * i S\left(t, q_{\Delta}, \xi_{\Delta}\right)=\mathcal{F}\left(t, \tau_{\nu-1}\right) \mathcal{F}\left(\tau_{\nu-1}, \tau_{\nu-2}\right) \cdots \mathcal{F}\left(\tau_{1}, 0\right)
$$

For we have

$$
\begin{aligned}
\mathcal{F}\left(\tau_{j+1}, \tau_{j}\right) & =\exp i \int_{\tau_{j}}^{\tau_{j+1}}\left\{\xi^{(j)} \cdot \dot{q}_{\Delta}(\theta)+\dot{q}_{\Delta}(\theta) \cdot A\left(\theta, q_{\Delta}(\theta)\right)-V\left(\theta, q_{\Delta}(\theta)\right)\right\} d \theta \\
& \times \exp -i\left(\tau_{j+1}-\tau_{j}\right)\left(c \widehat{\alpha} \cdot \xi^{(j)}+\widehat{\beta} m c^{2}\right)
\end{aligned}
$$

from (1.4). Hence we obtain (2.10) from (1.6).

In [20] we determined the Feynman path integral for the Pauli equation. There, to define the corresponding to $\exp * i S\left(t, q_{\Delta}, \xi_{\Delta}\right)$ we introduced the similar equation (1.11) in [20] to (2.9).

\section{$3 \quad$ Stability of $G(t, s)$}

Hereafter, where no confusion can arise, we write $\mathcal{S}^{N}, L^{2}\left(R^{d}\right)^{N}$ and $B_{M}^{a}\left(R^{d}\right)^{N}$ as $\mathcal{S}, L^{2}\left(R^{d}\right)$ and $B_{M}^{a}\left(R^{d}\right)$, respectively for the sake of simplicity, omitting the superscript $N$.

Lemma 3.1. Let $A(w)\left(w \in R^{d}\right)$ be an $N \times N$ matrix whose all components are continuously differentiable with respect to $w$. Then we have

$$
\frac{\partial}{\partial w_{j}} e^{A(w)}=\int_{0}^{1} e^{(1-\tau) A(w)} \frac{\partial A}{\partial w_{j}}(w) e^{\tau A(w)} d \tau .
$$


Proof. We set $u(t ; w)=e^{t A(w)}$. Then

$$
\frac{\partial u}{\partial t}(t ; w)=A(w) u(t ; w)
$$

So

$$
\frac{d}{d t} \frac{\partial u}{\partial w_{j}}(t ; w)=A(w) \frac{\partial u}{\partial w_{j}}(t ; w)+\frac{\partial A}{\partial w_{j}}(w) u(t ; w)
$$

with $\partial u(0 ; w) / \partial w_{j}=0$. Consequently we have

$$
\frac{\partial u}{\partial w_{j}}(t ; w)=\int_{0}^{t} e^{(t-\tau) A(w)} \frac{\partial A}{\partial w_{j}}(w) e^{\tau A(w)} d \tau,
$$

which shows (3.1).

Let $G_{\epsilon}(t, s)$ be the operator defined by (1.11).

Proposition 3.2. We assume (2.7) and that there exists a positive integer $M^{\prime}$ satisfying

$$
\left|\partial_{x}^{\alpha} A_{j}(t, x)\right| \leq C_{\alpha}<x>^{M^{\prime}}, \quad|\alpha| \geq 1
$$

in $[0, T] \times R^{d}$ for $j=1,2, \ldots, d$. Then $\left\{G_{\epsilon}(t, s)\right\}_{0<\epsilon \leq 1}$ is a bounded family of operators from $\mathcal{S}$ into itself and there exists an operator $G(t, s)$ on $\mathcal{S}$ independent of the choice of $\chi$ such that we have (1.13) in $\mathcal{S}$ for all $f \in \mathcal{S}$.

Proof. We write $S(t, s ; x, \xi, y)$ defined by $(1.10)$ as $(x-y) \cdot \xi+S_{0}(t, s ; x, \xi, y)$. Let $\alpha$ and $\beta$ be arbitrary multi-indices, and $l_{0}$ and $l_{1}$ arbitrary positive integers. Let $f \in \mathcal{S}$ and $\Delta_{x}=\sum_{j=1}^{d} \partial_{x_{j}}^{2}$. Then we have

$$
\begin{aligned}
& \iint e^{i S(t, s ; x, \xi, y)} x^{\alpha} \xi^{\beta} f(y) \chi(\epsilon \xi) d y d \xi=\iint e^{i x \cdot \xi}<x>^{-2 l_{0}} x^{\alpha}\left(1-\Delta_{\xi}\right)^{l_{0}} \\
& \times\left\{e^{-i y \cdot \xi} \xi^{\beta}<\xi>^{-2 l_{1}}\left(1-\Delta_{y}\right)^{l_{1}} e^{i S_{0}(t, s ; x, \xi, y)} f(y) \chi(\epsilon \xi)\right\} d y d \xi .
\end{aligned}
$$

Let $l_{0}$ and $l_{1}$ be large and note that $\widehat{\alpha}^{(j)}(j=1,2, \ldots, d)$ and $\widehat{\beta}$ are Hermitian matrices. Then we can prove from the assumptions of Proposition 3.2 in terms 
of Lemma 3.1 that $\left\{G_{\epsilon}(t, s)\right\}_{0<\epsilon \leq 1}$ is a bounded family of operators from $\mathcal{S}$ into itself and that as $\epsilon \rightarrow 0$, this converges to a bounded function

$$
\begin{aligned}
& \iint e^{i x \cdot \xi}<x>^{-2 l_{0}} x^{\alpha}\left(1-\Delta_{\xi}\right)^{l_{0}}\left\{e^{-i y \cdot \xi} \xi^{\beta}\right. \\
& \left.\times<\xi>^{-2 l_{1}}\left(1-\Delta_{y}\right)^{l_{1}} e^{i S_{0}(t, s ; x, \xi, y)} f(y)\right\} d y d \xi
\end{aligned}
$$

uniformly in $R_{x}^{d}$. In the same way we can easily complete the proof.

The main theorem in this section is the following.

Theorem 3.3. Let $G(t, s)$ be the operator on $\mathcal{S}$ defined in Proposition 3.2. Assume (2.1), (2.2) and (2.6). Then we have: (1) $G(t, s)$ can be extended to a bounded operator on $L^{2}$. (2) There exists a constant $K \geq 0$ such that (1.14) holds for all $f \in L^{2}$ and $0 \leq s, t \leq T$.

Leu us write $\mathbf{x}=(t, x) \in R^{d+1}$ and

$$
\mathbf{q}_{x, y}^{t, s}: \mathbf{q}_{x, y}^{t, s}(\theta)=\left(\theta, q_{x, y}^{t, s}(\theta)\right) \in R^{d+1}(s \leq \theta \leq t \text { or } t \leq \theta \leq s)
$$

Lemma 3.4. We have

$$
\left(\int_{\mathbf{q}_{y, x}^{t, s}}-\int_{\mathbf{q}_{y, z}^{t, s}}\right)(A \cdot d x-V d t)=(x-z) \cdot \Psi(t, s ; x, y, z),
$$

where $\Psi=\left(\Psi_{1}, \ldots, \Psi_{d}\right) \in R^{d}$ and

$$
\begin{aligned}
& \Psi_{j}(t, s ; x, y, z)=-\int_{0}^{1} A_{j}(s, z+\theta(x-z)) d \theta \\
& +(t-s) \int_{0}^{1} \int_{0}^{1} \sigma_{1} E_{j}\left(t-\sigma_{1}(t-s), y+\sigma_{1}(z-y)+\sigma_{1} \sigma_{2}(x-z)\right) d \sigma_{1} d \sigma_{2} \\
& +\sum_{k=1}^{d}\left(y_{k}-z_{k}\right) \int_{0}^{1} \int_{0}^{1} B_{j k}\left(t-\sigma_{1}(t-s), y+\sigma_{1}(z-y)+\sigma_{1} \sigma_{2}(x-z)\right) d \sigma_{1} d \sigma_{2} .
\end{aligned}
$$


Proof. The Stokes theorem shows

$$
\left(\int_{\mathbf{q}_{y, x}^{t, s}}-\int_{\mathbf{q}_{y, z}^{t, s}}+\int_{\mathbf{q}_{x, z}^{s, s}}\right)(A \cdot d x-V d t)=\iint_{\Lambda} d(A \cdot d x-V d t),
$$

where $\Lambda$ is the 2-dimensional plane with oriented boundary consisting of $\mathbf{q}_{y, x}^{t, s},-\mathbf{q}_{y, z}^{t, s}$ and $\mathbf{q}_{x, z}^{s, s}$. We introduce coordinates $\sigma=\left(\sigma_{1}, \sigma_{2}\right)\left(0 \leq \sigma_{1}, \sigma_{2} \leq 1\right)$ with the positive orientation in $\Lambda$ by

$$
\begin{aligned}
& (\tau(\sigma), \zeta(\sigma)) \\
& =\sigma_{2}\left\{\sigma_{1}(s, x)+\left(1-\sigma_{1}\right)(t, y)\right\}+\left(1-\sigma_{2}\right)\left\{\sigma_{1}(s, z)+\left(1-\sigma_{1}\right)(t, y)\right\} \\
& =\left(t-\sigma_{1}(t-s), y+\sigma_{1}(z-y)+\sigma_{1} \sigma_{2}(x-z)\right) \in R^{d+1}
\end{aligned}
$$

Since

$$
d(A \cdot d x-V d t)=-\sum_{j=1}^{d} E_{j}(t, x) d t \wedge d x_{j}+\sum_{1 \leq j<k \leq d} B_{j k} d x_{j} \wedge d x_{k}
$$

holds from (1.1), Lemma 3.4 can be proved from (3.5) as in the proof of Lemma 3.2 in $[16]$.

To avoid the complexity we suppose hereafter that $\chi$ in (1.11) is real-valued.

Proposition 3.5. Let $\Psi=\Psi(t, s ; x, y, z)$ be the function defined by (3.4). Then for $f \in \mathcal{S}$ we have

$$
\begin{aligned}
& \left(G_{\epsilon}(t, s)^{*} G_{\epsilon}(t, s) f\right)(x)=\iint e^{i(x-z) \cdot \xi} d z d \xi \iint e^{-i \eta \cdot w} e^{i(t-s)\left(c \widehat{\alpha} \cdot \xi+c \widehat{\alpha} \cdot \Psi+\widehat{\beta} m c^{2}\right)} \\
& \times e^{-i(t-s)\left(c \widehat{\alpha} \cdot \xi+c \widehat{\alpha} \cdot \Psi+\widehat{\beta} m c^{2}-c \widehat{\alpha} \cdot \eta\right)} \chi(\epsilon(\xi+\Psi)) \chi(\epsilon(\xi+\Psi-\eta)) f(z) d u d \eta
\end{aligned}
$$

with $\Psi=\Psi(t, s ; x, w+z, z)$, where $\eta \in R^{d}, w \in R^{d}$ and $G_{\epsilon}(t, s)^{*}$ denotes the formally adjoint operator of $G_{\epsilon}(t, s)$. 
Proof. Since $S(t, s ; x, \xi, y)$ is a Hermitian matrix from $(1.10), G_{\epsilon}(t, s)^{*}$ is written by

$$
\left(G_{\epsilon}(t, s)^{*} f\right)(x)=\iint e^{-i S(t, s ; y, \xi, x)} f(y) \chi(\epsilon \xi) d y d \xi
$$

from (1.11). So from (1.10) we have

$$
\begin{aligned}
& \left(G_{\epsilon}(t, s)^{*} G_{\epsilon}(t, s) f\right)(x)=\iint\left[\exp -i\left\{\xi \cdot(y-x)+\int_{\mathbf{q}_{y, x}^{t, s}}(A \cdot d x-V d t)\right.\right. \\
& \left.\left.-(t-s)\left(c \widehat{\alpha} \cdot \xi+\widehat{\beta} m c^{2}\right)\right\}\right] \chi(\epsilon \xi) d y d \xi \iint\left[\operatorname { e x p } i \left\{\eta \cdot(y-z)+\int_{\mathbf{q}_{y}^{t, s,}}(A \cdot d x-V d t)\right.\right. \\
& \left.\left.-(t-s)\left(c \widehat{\alpha} \cdot \eta+\widehat{\beta} m c^{2}\right)\right\}\right] \chi(\epsilon \eta) f(z) d z d \eta=\iint e^{i(x-z) \cdot \xi} e^{i(t-s)\left(c \widehat{\alpha} \cdot \xi+\widehat{\beta} m c^{2}\right)} \\
& \quad \times \chi(\epsilon \xi) d z d \xi \iint e^{-i(\xi-\eta) \cdot(y-z)}\left\{\exp -i\left(\int_{\mathbf{q}_{y, x}^{t, s}}-\int_{\mathbf{q}_{y, z}^{t, s}}\right)(A \cdot d x-V d t)\right\} \\
& \quad \times e^{-i(t-s)\left(c \widehat{\alpha} \cdot \eta+\widehat{\beta} m c^{2}\right)} \chi(\epsilon \eta) f(z) d y đ \eta .
\end{aligned}
$$

Making the change of variables by $\eta^{\prime}=\xi-\eta: \eta \rightarrow \eta^{\prime}$ and $w=y-z: y \rightarrow w$ and using (3.3), we get

$$
\begin{aligned}
& \left(G_{\epsilon}(t, s)^{*} G_{\epsilon}(t, s) f\right)(x)=\iint e^{i(x-z) \cdot \xi} e^{i(t-s)\left(c \widehat{\alpha} \cdot \xi+\widehat{\beta} m c^{2}\right)} \chi(\epsilon \xi) d z d \xi \iint e^{-i \eta^{\prime} \cdot w} \\
& \times e^{-i(x-z) \cdot \Psi} e^{-i(t-s)\left(c \widehat{\alpha} \cdot \xi+\widehat{\beta} m c^{2}-c \widehat{\alpha} \cdot \eta^{\prime}\right)} \chi\left(\epsilon\left(\xi-\eta^{\prime}\right)\right) f(z) d u \tau \eta^{\prime}
\end{aligned}
$$

with $\Psi=\Psi(t, s ; x, w+z, z)$. Making the change of variables by $\xi^{\prime}=\xi-$ $\Psi(t, s ; x, w+z, z): \xi \rightarrow \xi^{\prime}$, we obtain (3.6).

Proposition 3.6. Under the assumptions of Theorem 3.3 we have

$$
\left|\partial_{x}^{\alpha} \partial_{y}^{\beta} \partial_{z}^{\gamma} \Psi_{j}(t, s ; x, y, z)\right| \leq C_{\alpha, \beta, \gamma},|\alpha+\beta+\gamma| \geq 1
$$

in $0 \leq s, t \leq T$ and $x, y, z \in R^{d}$ for $j=1,2, \ldots, d$.

Proof. It follows from (2.6) and (2.1) that the first term and the second one in (3.4) satisfy the relations (3.8), respectively. We can easily see from (2.2) that 
$\partial_{x}^{\alpha} B_{j k}(t, x)$ for all $\alpha$ are bounded in $[0, T] \times R^{d}$. So, using (ii) of Lemma 3.5 in [16], we can see from (2.2) that the third term in (3.4) also satisfies (3.8).

Lemma 3.7. Let $A$ and $B$ be $N \times N$ matrices. Then we have

$$
e^{A+B}=e^{A}+\int_{0}^{1} d \theta \int_{0}^{1} e^{(1-\tau)(A+\theta B)} B e^{\tau(A+\theta B)} d \tau
$$

Proof. Set $u(\theta)=e^{A+\theta B}(\theta \in R)$. Then we have

$$
\frac{\partial u}{\partial \theta}(\theta)=\int_{0}^{1} e^{(1-\tau)(A+\theta B)} B e^{\tau(A+\theta B)} d \tau
$$

from (3.1). Because of

$$
e^{A+B}-e^{A}=u(1)-u(0)=\int_{0}^{1} \frac{\partial u}{\partial \theta}(\theta) d \theta
$$

(3.9) follows from this.

Let us prove Theorem 3.3. Let $f \in \mathcal{S}$. In terms of (3.9) we can write

$$
\begin{aligned}
& e^{-i(t-s)\left(c \widehat{\alpha} \cdot \xi+c \widehat{\alpha} \cdot \Psi+\widehat{\beta} m c^{2}-c \widehat{\alpha} \cdot \eta\right)}=e^{-i(t-s)\left(c \widehat{\alpha} \cdot \xi+c \widehat{\alpha} \cdot \Psi+\widehat{\beta} m c^{2}\right)} \\
& +\int_{0}^{1} d \theta \int_{0}^{1} d \tau e^{-i(t-s)(1-\tau)\left(c \widehat{\alpha} \cdot \xi+c \widehat{\alpha} \cdot \Psi+\widehat{\beta} m c^{2}-\theta c \widehat{\alpha} \cdot \eta\right)} \\
& \times i(t-s) c \widehat{\alpha} \cdot \eta e^{-i(t-s) \tau\left(c \widehat{\alpha} \cdot \xi+c \widehat{\alpha} \cdot \Psi+\widehat{\beta} m c^{2}-\theta c \widehat{\alpha} \cdot \eta\right)}
\end{aligned}
$$

Applying this to (3.6), we get

$$
\begin{aligned}
& \left(G_{\epsilon}(t, s)^{*} G_{\epsilon}(t, s) f\right)(x)=\iint e^{i(x-z) \cdot \xi} f(z) d z d \xi \iint e^{-i \eta \cdot w} \chi(\epsilon(\xi+\Psi)) \\
& \times \chi(\epsilon(\xi+\Psi-\eta)) d u \Uparrow \eta+c(t-s) \iint e^{i(x-z) \cdot \xi} d z d \xi \iint e^{-i \eta \cdot w} e^{i(t-s)\left(c \widehat{\alpha} \cdot \xi+c \widehat{\alpha} \cdot \Psi+\widehat{\beta} m c^{2}\right)} \\
& \times\left\{\int_{0}^{1} d \theta \int_{0}^{1} d \tau e^{-i(t-s)(1-\tau)\left(c \widehat{\alpha} \cdot \xi+c \widehat{\alpha} \cdot \Psi+\widehat{\beta} m c^{2}-\theta c \widehat{\alpha} \cdot \eta\right)} i \widehat{\alpha} \cdot \eta e^{-i(t-s) \tau\left(c \widehat{\alpha} \cdot \xi+c \widehat{\alpha} \cdot \Psi+\widehat{\beta} m c^{2}-\theta c \widehat{\alpha} \cdot \eta\right)}\right\} \\
& \quad \times \chi(\epsilon(\xi+\Psi)) \chi(\epsilon(\xi+\Psi-\eta)) f(z) d u \pi \eta \eta
\end{aligned}
$$


Consequently we obtain

$$
\begin{aligned}
& \left(G_{\epsilon}(t, s)^{*} G_{\epsilon}(t, s) f\right)(x)=\iint e^{i(x-z) \cdot \xi} f(z) d z d \xi \iint e^{-i \eta \cdot w} \chi(\epsilon(\xi+\Psi)) \\
& \times \chi(\epsilon(\xi+\Psi-\eta)) d u d \eta+c(t-s) \iint e^{i(x-z) \cdot \xi} d z d \xi \int_{0}^{1} d \theta \int_{0}^{1} d \tau \\
& \times \iint e^{i(t-s)\left(c \widehat{\alpha} \cdot \xi+c \widehat{\alpha} \cdot \Psi+\widehat{\beta} m c^{2}\right)} e^{-i(t-s)(1-\tau)\left(c \widehat{\alpha} \cdot \xi+c \widehat{\alpha} \cdot \Psi+\widehat{\beta} m c^{2}-\theta c \widehat{\alpha} \cdot \eta\right)} e^{-i \eta \cdot w} i \widehat{\alpha} \cdot \eta \\
& \times e^{-i(t-s) \tau\left(c \widehat{\alpha} \cdot \xi+c \widehat{\alpha} \cdot \Psi+\widehat{\beta} m c^{2}-\theta c \widehat{\alpha} \cdot \eta\right)} \chi(\epsilon(\xi+\Psi)) \chi(\epsilon(\xi+\Psi-\eta)) f(z) d u d \eta,
\end{aligned}
$$

where $\Psi=\Psi(t, s ; x, w+z, z)$.

Let us consider the first term in (3.10). Let $l_{0}$ be an integer such that $2 l_{0}>d$. Then we can write

$$
\begin{aligned}
& \iint e^{-i \eta \cdot w} \chi(\epsilon(\xi+\Psi)) \chi(\epsilon(\xi+\Psi-\eta)) d u d \eta=\iint e^{-i \eta \cdot w}<\eta>^{-2 l_{0}}\left(1-\Delta_{w}\right)^{l_{0}} \\
& \cdot\left\{<w>^{-2 l_{0}}\left(1-\Delta_{\eta}\right)^{l_{0}}\right\} \chi(\epsilon(\xi+\Psi)) \chi(\epsilon(\xi+\Psi-\eta)) d u \varpi \eta .
\end{aligned}
$$

Noting (3.8), we can see as in the proof of Proposition 3.2 that as $\epsilon \rightarrow 0$, the first term in (3.10) converges to $\lim _{\epsilon \rightarrow 0} \iint e^{i(x-z) \cdot \xi} f(z) d z d \xi \iint e^{-i \eta \cdot w} \chi(\epsilon \eta) \chi(\epsilon w) d u \pi \eta$ $=f(x)$ in $\mathcal{S}$.

Let us consider the second term in (3.10). Noting $\left(-\partial_{w_{j}}\right) e^{-i \eta \cdot w}=i \eta_{j} e^{-i \eta \cdot w}$, we first integrate by parts with respect to $w$. Next we use Lemma 3.1, (3.8) and the fact that $\widehat{\alpha}^{(j)}(j=1,2, \ldots, d)$ and $\widehat{\beta}$ are Hermitian and integrate by parts with respect to $w$ and $\eta$ as in the proof of the first term. Then we can prove that as $\epsilon \rightarrow 0$, the second term converges to a pseudo-differential operator $(t-s)^{2} P(t, s) f=(t-s)^{2} P\left(t, s ; X, D_{X}, X^{\prime}\right) f:=\iint e^{i(x-z) \cdot \xi} p(x, \xi, z) f(z) d z d \xi$ in $\mathcal{S}$, where the symbol $p_{i j}(t, s ; x, \xi, z)(i, j=1,2, \ldots, N)$ of the $(i, j)$-component of $P(t, s)$ satisfies

$$
\left|\partial_{\xi}^{\alpha} \partial_{x}^{\beta} \partial_{z}^{\gamma} p_{i j}(t, s ; x, \xi, z)\right| \leq C_{\alpha, \beta, \gamma}, 0 \leq s, t \leq T
$$


in $R^{3 d}$ for all $\alpha, \beta$ and $\gamma$. Consequently we get

$$
\lim _{\epsilon \rightarrow 0} G_{\epsilon}(t, s)^{*} G_{\epsilon}(t, s) f=f+(t-s)^{2} P(t, s) f
$$

in $\mathcal{S}$, which shows

$$
G(t, s)^{*} G(t, s) f=f+(t-s)^{2} P(t, s) f
$$

from Proposition 3.2. Hence we obtain

$$
\begin{aligned}
& \|G(t, s) f\|^{2}=(f, f)+(t-s)^{2}(P(t, s) f, f) \\
& \leq\|f\|^{2}+2 K(t-s)^{2}\|f\|^{2} \leq e^{2 K(t-s)^{2}}\|f\|^{2}
\end{aligned}
$$

for $f \in \mathcal{S}$ with a constant $K \geq 0$ from the Calderón-Vaillancourt theorem (cf. [2], Theorem 1.6, p.224 of [23]). Thus we could complete the proof of Theorem 3.3 .

\section{Consistency of $G(t, s)$}

Let $G_{\epsilon}(t, s)$ be the operator defined by (1.11).

Proposition 4.1. Let $H(t)$ be the Dirac operator defined by (1.2). Then for $f \in \mathcal{S}$ we have

$$
\begin{aligned}
& {\left[i \frac{\partial}{\partial t}-H(t)\right] G_{\epsilon}(t, s) f=R_{\epsilon}(t, s) f} \\
& :=\iint r(t, s ; x, y) e^{i S(t, s ; x, \xi, y)} f(y) \chi(\epsilon \xi) d y đ \xi
\end{aligned}
$$


where

$$
\begin{aligned}
& r(t, s ; x, y)=(x-y) \cdot \int_{0}^{1}(1-\theta) E(t-\theta(t-s), x-\theta(x-y)) d \theta \\
& -c \sum_{j=1}^{d} \widehat{\alpha}^{(j)}\left[\int_{0}^{1}\left\{A_{j}(t-\theta(t-s), x-\theta(x-y))-A_{j}(t, x)\right\} d \theta\right. \\
& +(x-y) \cdot \int_{0}^{1}(1-\theta) \frac{\partial A}{\partial x_{j}}(t-\theta(t-s), x-\theta(x-y)) d \theta \\
& \left.-(t-s) \int_{0}^{1}(1-\theta) \frac{\partial V}{\partial x_{j}}(t-\theta(t-s), x-\theta(x-y)) d \theta\right] .
\end{aligned}
$$

Proof. We sometimes write $\rho=t-s$. The classical action $S(t, s ; x, \xi, y)$ defined by (1.10) can be written as

$$
\begin{aligned}
& S(t, s ; x, \xi, y)=(x-y) \cdot \xi+(x-y) \cdot \int_{0}^{1} A(t-\theta(t-s), x-\theta(x-y)) d \theta \\
& \quad-\int_{s}^{t} V\left(\theta, y+\frac{\theta-s}{t-s}(x-y)\right) d \theta-(t-s)\left(c \widehat{\alpha} \cdot \xi+\widehat{\beta} m c^{2}\right) \\
& =(x-y) \cdot \xi+(x-y) \cdot \int_{0}^{1} A(t-\theta \rho, x-\theta(x-y)) d \theta \\
& \quad-(t-s) \int_{0}^{1} V(t-\theta \rho, x-\theta(x-y)) d \theta-(t-s)\left(c \widehat{\alpha} \cdot \xi+\widehat{\beta} m c^{2}\right) .
\end{aligned}
$$

So we have

$$
\begin{aligned}
& \partial_{t} S(t, s ; x, \xi, y)=(x-y) \cdot \int_{0}^{1}(1-\theta) \frac{\partial A}{\partial t}(t-\theta \rho, x-\theta(x-y)) d \theta-V(t, x) \\
& +\frac{1}{t-s} \int_{s}^{t} \frac{\theta-s}{t-s}(x-y) \cdot \frac{\partial V}{\partial x}\left(\theta, y+\frac{\theta-s}{t-s}(x-y)\right) d \theta-\left(c \widehat{\alpha} \cdot \xi+\widehat{\beta} m c^{2}\right) \\
& =(x-y) \cdot \int_{0}^{1}(1-\theta) \frac{\partial A}{\partial t}(t-\theta \rho, x-\theta(x-y)) d \theta-V(t, x) \\
& +(x-y) \cdot \int_{0}^{1}(1-\theta) \frac{\partial V}{\partial x}(t-\theta \rho, x-\theta(x-y)) d \theta-\left(c \widehat{\alpha} \cdot \xi+\widehat{\beta} m c^{2}\right),
\end{aligned}
$$


which shows

$$
\begin{aligned}
& \partial_{t} S(t, s ; x, \xi, y)=-V(t, x)-\left(c \widehat{\alpha} \cdot \xi+\widehat{\beta} m c^{2}\right) \\
& -(x-y) \cdot \int_{0}^{1}(1-\theta) E(t-\theta \rho, x-\theta(x-y)) d \theta
\end{aligned}
$$

from (1.1). We should note

$$
\frac{\partial}{\partial t} e^{i S(t, s ; x, \xi, y)}=i \frac{\partial S}{\partial t} e^{i S(t, s ; x, \xi, y)}=i e^{i S(t, s ; x, \xi, y)} \frac{\partial S}{\partial t}
$$

because the matrix term in $S$ is only $-(t-s)\left(c \widehat{\alpha} \cdot \xi+\widehat{\beta} m c^{2}\right)$. From (4.3) we have

$$
\begin{aligned}
& \partial_{x_{j}} S(t, s ; x, \xi, y)=\xi_{j}+\int_{0}^{1} A_{j}(t-\theta \rho, x-\theta(x-y)) d \theta \\
& +(x-y) \cdot \int_{0}^{1}(1-\theta) \frac{\partial A}{\partial x_{j}}(t-\theta \rho, x-\theta(x-y)) d \theta \\
& \quad-\rho \int_{0}^{1}(1-\theta) \frac{\partial V}{\partial x_{j}}(t-\theta \rho, x-\theta(x-y)) d \theta
\end{aligned}
$$

and so

$$
\begin{aligned}
& \partial_{x_{j}} S(t, s ; x, \xi, y)-A_{j}(t, x)=\xi_{j}+\int_{0}^{1}\left\{A_{j}(t-\theta \rho, x-\theta(x-y))\right. \\
& \left.-A_{j}(t, x)\right\} d \theta+(x-y) \cdot \int_{0}^{1}(1-\theta) \frac{\partial A}{\partial x_{j}}(t-\theta \rho, x-\theta(x-y)) d \theta \\
& \quad-\rho \int_{0}^{1}(1-\theta) \frac{\partial V}{\partial x_{j}}(t-\theta \rho, x-\theta(x-y)) d \theta .
\end{aligned}
$$

We also note

$$
\frac{\partial}{\partial x_{j}} e^{i S(t, s ; x, \xi, y)}=i \frac{\partial S}{\partial x_{j}} e^{i S(t, s ; x, \xi, y)}=i e^{i S(t, s ; x, \xi, y)} \frac{\partial S}{\partial x_{j}} .
$$


From (4.4)-(4.7) we have

$$
\begin{aligned}
& {\left[i \frac{\partial}{\partial t}-H(t)\right] e^{i S(t, s ; x, \xi, y)}=\left[i \frac{\partial}{\partial t}-\left\{c \sum_{j=1}^{d} \widehat{\alpha}^{(j)}\left(\frac{1}{i} \frac{\partial}{\partial x_{j}}-A_{j}(t, x)\right)\right.\right.} \\
& \left.\left.+\widehat{\beta} m c^{2}+V(t, x)\right\}\right] e^{i S}=-\left\{\frac{\partial S}{\partial t}+c \sum_{j=1}^{d} \widehat{\alpha}^{(j)}\left(\partial_{x_{j}} S-A_{j}\right)+\widehat{\beta} m c^{2}\right. \\
& +V(t, x)\} e^{i S}=-\left[\frac{\partial S}{\partial t}+c \sum_{j=1}^{d} \widehat{\alpha}^{(j)}\left\{\xi_{j}+\int_{0}^{1}\left(A_{j}(t-\theta \rho, x-\theta(x-y))\right.\right.\right. \\
& \left.-A_{j}(t, x)\right) d \theta+(x-y) \cdot \int_{0}^{1}(1-\theta) \frac{\partial A}{\partial x_{j}}(t-\theta \rho, x-\theta(x-y)) d \theta \\
& \left.\left.-\rho \int_{0}^{1}(1-\theta) \frac{\partial V}{\partial x_{j}}(t-\theta \rho, x-\theta(x-y)) d \theta\right\}+\widehat{\beta} m c^{2}+V(t, x)\right] e^{i S} \\
& =\left[(x-y) \cdot \int_{0}^{1}(1-\theta) E(t-\theta \rho, x-\theta(x-y)) d \theta\right. \\
& -c \sum_{j=1}^{d} \widehat{\alpha}^{(j)}\left\{\int_{0}^{1}\left(A_{j}(t-\theta \rho, x-\theta(x-y))-A_{j}(t, x)\right) d \theta\right. \\
& +(x-y) \cdot \int_{0}^{1}(1-\theta) \frac{\partial A}{\partial x_{j}}(t-\theta \rho, x-\theta(x-y)) d \theta \\
& \left.\left.-\rho \int_{0}^{1}(1-\theta) \frac{\partial V}{\partial x_{j}}(t-\theta \rho, x-\theta(x-y)) d \theta\right\}\right] e^{i S},
\end{aligned}
$$

which shows (4.2).

TheOREm 4.2. Let $R_{\epsilon}(t, s)$ be the operator defined by (4.1). Then under the assumptions of Theorem 2.2 there exists an operator $R(t, s)$ independent of the choice of $\chi$ on $\mathcal{S}$ such that

$$
\lim _{\epsilon \rightarrow 0} R_{\epsilon}(t, s) f=R(t, s) f
$$

in $\mathcal{S}$ for all $f \in \mathcal{S}$. We also have

$$
\|R(t, s) f\| \leq C|t-s|\left\|<\cdot>^{M} f\right\|, 0 \leq s, t \leq T
$$

for $f \in \mathcal{S}$ with a constant $C \geq 0$. 
Proof. Since we assume (2.1), (2.6) and (2.7), we can prove (4.8) in $\mathcal{S}$ for $f \in \mathcal{S}$ from (4.1) and (4.2) as in the proof of Proposition 3.2.

We note that $r(t, s ; x, y)$ defined by $(4.2)$ is a Hermitian matrix. So as in the proof of (3.7) we have

$$
\left(R_{\epsilon}(t, s)^{*} f\right)(x)=\iint e^{-i S(t, s ; y, \xi, x)} r(t, s ; y, x) f(y) \chi(\epsilon \xi) d y d \xi
$$

Hence we can prove

$$
\begin{aligned}
& \left(R_{\epsilon}(t, s)^{*} R_{\epsilon}(t, s) f\right)(x)=\iint e^{-i S(t, s ; y, \xi, x)} r(t, s ; y, x) \chi(\epsilon \xi) d y d \xi \\
& \times \iint r(t, s ; y, z) e^{i S(t, s ; y, \eta, z)} f(z) \chi(\epsilon \eta) d z d \eta=\iint e^{i(x-z) \cdot \xi} d z d \xi \\
& \times \iint e^{-i \eta \cdot w} e^{i(t-s)\left(c \widehat{\alpha} \cdot \xi+c \widehat{\alpha} \cdot \Psi+\widehat{\beta} m c^{2}\right)} r(t, s ; w+z, x) r(t, s ; w+z, z) \\
& \times e^{-i(t-s)\left(c \widehat{\alpha} \cdot \xi+c \widehat{\alpha} \cdot \Psi+\widehat{\beta} m c^{2}-c \widehat{\alpha} \cdot \eta\right)} \chi(\epsilon(\xi+\Psi)) \chi(\epsilon(\xi+\Psi-\eta)) f(z) d u \varpi \hbar \eta
\end{aligned}
$$

with $\Psi=\Psi(t, s ; x, w+z, z)$ as in the proof of (3.6).

We can write (4.2) as

$$
\begin{aligned}
& r(t, s ; x, y)=(x-y) \cdot \int_{0}^{1}(1-\theta) E(t-\theta \rho, x-\theta(x-y)) d \theta \\
& +c \sum_{j=1}^{d} \widehat{\alpha}^{(j)}\left\{(t-s) \int_{0}^{1} \theta d \theta \int_{0}^{1} \frac{\partial A_{j}}{\partial t}\left(t-\theta^{\prime} \theta \rho, x-\theta(x-y)\right) d \theta^{\prime}\right. \\
& +(x-y) \cdot \int_{0}^{1} \theta d \theta \int_{0}^{1} \frac{\partial A_{j}}{\partial x}\left(t, x-\theta^{\prime} \theta(x-y)\right) d \theta^{\prime} \\
& -(x-y) \cdot \int_{0}^{1}(1-\theta) \frac{\partial A}{\partial x_{j}}(t-\theta \rho, x-\theta(x-y)) d \theta \\
& \left.+(t-s) \int_{0}^{1}(1-\theta) \frac{\partial V}{\partial x_{j}}(t-\theta \rho, x-\theta(x-y)) d \theta\right\} .
\end{aligned}
$$

Let us apply (4.12) to $r(t, s ; w+z, x)$ and $r(t, s ; w+z, z)$ in (4.11). Noting $w+z-x=w-(x-z), w+z-z=w, i w_{j} e^{-i \eta \cdot w}=-\partial_{\eta_{j}} e^{-i \eta \cdot w}$ and $i\left(x_{j}-\right.$ 
$\left.z_{j}\right) e^{-i(x-z) \cdot \xi}=\partial_{\xi_{j}} e^{-i(x-z) \cdot \xi}$, we integrate by parts with respect to $\xi$ and $\eta$ in (4.11). Then from the assumptions of Theorem 4.2 we can prove

$$
\lim _{\epsilon \rightarrow 0} R_{\epsilon}(t, s)^{*} R_{\epsilon}(t, s) f=(t-s)^{2} Q(t, s) f
$$

as in the proof of $(3.12)$, where $Q(t, s)$ is a pseudo-differential operator with symbol $q_{i j}(t, s ; x, \xi, z)(i, j=1,2, \ldots, N)$ satisfying

$$
\left|\partial_{\xi}^{\alpha} \partial_{x}^{\beta} \partial_{z}^{\gamma} q_{i j}(t, s ; x, \xi, z)\right| \leq C_{\alpha, \beta, \gamma}\left(<x>^{2 M}+<z>^{2 M}\right), 0 \leq s, t \leq T
$$

in $R^{3 d}$ for all $\alpha, \beta$ and $\gamma$. Set

$\tilde{q}_{i j}(t, s ; x, \xi)=<x>^{-M}$ Os $-\iint e^{-i y \cdot \eta} q_{i j}(t, s ; x, \xi+\eta, x+y)<x+y>^{-M} d y d \eta$

Then from (4.14) we have

$$
\left|\partial_{\xi}^{\alpha} \partial_{x}^{\beta} \tilde{q}_{i j}(t, s ; x, \xi)\right| \leq C_{\alpha, \beta}, 0 \leq s, t \leq T
$$

in $R^{2 d}$ for all $\alpha$ and $\beta$ as in the proof of Proposition 3.2, which shows

$$
\left\|\widetilde{Q}_{i j}\left(t, s ; X, D_{x}\right) f\right\| \leq C\|f\|, 0 \leq s, t \leq T
$$

for $f \in L^{2}$ with a constant $C \geq 0$ from the Calderón-Vaillancourt theorem. We also have

$$
Q(t, s) f=<\cdot>^{M} \widetilde{Q}(t, s)\left(<\cdot>^{M} f\right)
$$

from Theorem 2.5, p.73 of [23]. Hence from (4.8) and (4.13) we get

$$
\begin{aligned}
& \|R(t, s) f\|^{2}=(t-s)^{2}(Q(t, s) f, f) \\
& =(t-s)^{2}\left(<\cdot>^{M} \widetilde{Q}(t, s)\left(<\cdot>^{M} f\right), f\right) \leq C(t-s)^{2}\left\|<\cdot>^{M} f\right\|^{2}
\end{aligned}
$$

with another constant $C \geq 0$. Thus we could complete the proof. 
THEOREM 4.3. Under the assumptions of Theorem 2.2 we have

$$
\|R(t, s) f\|_{B_{M}^{a}} \leq C_{a}|t-s|\|f\|_{B_{M}^{a+1}}, 0 \leq s, t \leq T
$$

for $a=0,1,2, \ldots$ with constants $C_{a} \geq 0$.

Proof. For $a=0$ we proved (4.15) in Theorem 4.2. Let $a=1,2, \ldots$ We can easily have

$$
<x>^{a M} R_{\epsilon}(t, s) f=\iint<x>^{a M} r(t, s ; x, y) e^{i S(t, s ; x, \xi, y)} f(y) \chi(\epsilon \xi) d y d \xi
$$

from (4.1). So as in the proof of (4.9) we get

$$
\left\|<\cdot>^{a M} R(t, s) f\right\| \leq C_{a}|t-s|\left\|<\cdot>^{(a+1) M} f\right\|, 0 \leq s, t \leq T .
$$

Let $w \in R^{d}$ and set

$$
\begin{gathered}
\phi(t, s ; x, \xi, w):=-w \cdot \xi-w \cdot \int_{0}^{1} A(t-\theta \rho, x+\theta w) d \theta \\
-\rho \int_{0}^{1} V(t-\theta \rho, x+\theta w) d \theta-\rho\left(c \widehat{\alpha} \cdot \xi+\widehat{\beta} m c^{2}\right) .
\end{gathered}
$$

Then from (4.1) and (4.3) we can write

$$
\left(R_{\epsilon}(t, s) f\right)(x)=\iint r(t, s ; x, x+w) e^{i \phi(t, s ; x, \xi, w)} f(x+w) \chi(\epsilon \xi) d u d \xi .
$$

Let $\gamma$ be a multi-index such that $|\gamma|=a$. Then it follows from (4.12), (4.17) and (4.18) that

$$
\begin{gathered}
\partial_{x}^{\gamma}\left(R_{\epsilon}(t, s) f\right)=\sum_{\kappa \leq \gamma} R_{\kappa \epsilon}(t, s) \partial_{x}^{\kappa} f, \\
r_{\kappa}(t, s ; x, y)=w \cdot r_{\kappa}^{(1)}(t, s ; x, y)+(t-s) r_{\kappa}^{(2)}(t, s ; x, y), w=y-x,
\end{gathered}
$$

where $r_{\kappa}^{(1)} \in R^{d}$ and we have

$$
\left|\partial_{x}^{\alpha} \partial_{y}^{\beta} r_{\kappa}^{(j)}(t, s ; x, y)\right| \leq C_{\alpha, \beta}\left(<x>^{M}+<y>^{M}\right)^{|\gamma-\kappa|+1}, 0 \leq s, t \leq T
$$


for all $\alpha, \beta$ and $j=1,2$ from (2.1) and (2.6)-(2.8). Hence as in the proof of (4.9) we can see

$$
\left\|\partial_{x}^{\gamma}(R(t, s) f)\right\| \leq C_{a}|t-s| \sum_{\kappa \leq \gamma}\left\|<\cdot>^{M(|\gamma-\kappa|+1)} \partial_{x}^{\kappa} f\right\| .
$$

Because of

$$
s_{1} s_{2} \leq \frac{1}{p} s_{1}^{p}+\frac{1}{q} s_{2}^{q}, \quad \frac{1}{p}+\frac{1}{q}=1
$$

for $s_{1}, s_{2} \geq 0$ and $1<p$ it holds

$$
<x>^{M\left(k+1-k^{\prime}\right)}<\xi>^{k^{\prime}} \leq \frac{1}{p}<x>^{M(k+1)}+\frac{1}{q}<\xi>^{k+1}
$$

for integers $0 \leq k^{\prime} \leq k$ with some constants $p>1$ and $q=p /(1-p)(>1)$. It follows from Lemma 2.3 in [15] that there exist a constant $\mu_{a} \geq 0$ and a function $p_{a, M}(x, \xi)$ satisfying

$$
\left|\partial_{\xi}^{\alpha} \partial_{x}^{\beta} p_{a, M}(x, \xi)\right| \leq C_{\alpha, \beta}\left(1+<x>^{a M}+<\xi>^{a}\right)^{-1}
$$

in $R^{2 d}$ for all $\alpha$ and $\beta$, and

$$
P_{a, M}\left(x, D_{x}\right) f=\Lambda_{a, M}^{-1} f:=\left(\mu_{a}+<X>^{a M}+<D_{x}>^{a}\right)^{-1} f
$$

for $f \in \mathcal{S}$. Consequently we can easily see from (4.22) as in the proof of (4.9) that $<\cdot>^{M(|\gamma-\kappa|+1)} \partial_{x}^{\kappa} \cdot P_{a+1, M}\left(X, D_{x}\right)$ is a bounded operator on $L^{2}$. Hence from (4.21) and (4.24) we have

$$
\left\|\partial_{x}^{\gamma}(R(t, s) f)\right\| \leq C_{a}^{\prime}|t-s|\left\|\Lambda_{a+1, M} f\right\|
$$

which shows

$$
\left\|\partial_{x}^{\gamma}(R(t, s) f)\right\| \leq C_{a}|t-s|\|f\|_{B_{M}^{a+1}}
$$

for $|\gamma|=a$ with another constant $C_{a} \geq 0$ from Lemma 2.4 in [15]. Thus we can complete the proof together with (4.16). 
Corollary 4.4. Let $G(t, s)$ be the operator defined in Proposition 3.2. We assume (2.1), (2.2), (2.6) and (2.7). Then we have

$$
\|G(t, s) f\|_{B_{M}^{a}} \leq C_{a}\|f\|_{B_{M}^{a}}, 0 \leq s, t \leq T
$$

for $a=0,1,2, \ldots$ with constants $C_{a} \geq 0$.

Proof. We proved (4.26) in Theorem 3.3 for $a=0$. Let $a \geq 1$. We can easily see

$$
\left\|<\cdot>^{a M} G(t, s) f\right\| \leq C_{a}\left\|<\cdot>^{a M} f\right\|
$$

as in the proof of (4.16). We can also prove

$$
\left\|\partial_{x}^{\gamma} G(t, s) f\right\| \leq C_{a}\|f\|_{B_{M}^{a}}
$$

for $|\gamma|=a$ as in the proof of (4.25). Hence we can complete the proof.

\section{$5 \quad$ Proof of the main results}

Theorem 5.1. Let $G(t, s)$ be the operator defined in Proposition 3.2. We assume (2.1), (2.2), (2.6) and (2.7). Then, there exist constants $K_{a} \geq 0(a=$ $1,2, \ldots$ ) such that

$$
\|G(t, s) f\|_{B_{M}^{a}} \leq e^{K_{a}|t-s|}\|f\|_{B_{M}^{a}}, \quad 0 \leq s, t \leq T
$$

for $f \in \mathcal{S}$.

Proof. Letting $\phi(t, s ; x, \xi, w)$ be the function defined by (4.17), we can write

$$
\left(G_{\epsilon}(t, s) f\right)(x)=\iint e^{i \phi(t, s ; x, \xi, w)} f(x+w) \chi(\epsilon \xi) d w \hbar \xi
$$


from (1.11) and (4.3) as in the way of (4.18). Let $\gamma$ be a multi-index such that $|\gamma|=a M$. Then it holds

$$
\begin{aligned}
& x^{\gamma} G_{\epsilon}(t, s) f-G_{\epsilon}(t, s)\left(x^{\gamma} f\right)=-\iint e^{i \phi(t, s ; x, \xi, w)}\left\{(x+w)^{\gamma}-x^{\gamma}\right\} \\
& \times f(x+w) \chi(\epsilon \xi) d u d \xi=\iint e^{i \phi(t, s ; x, \xi, w)} w \cdot q(x, w) f(x+w) \chi(\epsilon \xi) d u d \xi \\
& =\iint e^{i S(t, s ; x, \xi, y)}(y-x) \cdot q(x, y-x) f(y) \chi(\epsilon \xi) d y d \xi
\end{aligned}
$$

where we have

$$
\left|\partial_{x}^{\alpha} \partial_{w}^{\beta} q_{j}(x, w)\right| \leq C_{\alpha, \beta}\left(<x>^{a M-1}+<w>^{a M-1}\right)
$$

for all $\alpha$ and $\beta$ and $j=1,2, \ldots, d$ as in the proof of (4.22). Consequently as in the proof of Theorem 4.2 we can prove

$$
\left\|x^{\gamma} G(t, s) f-G(t, s)\left(x^{\gamma} f\right)\right\| \leq C|t-s|\left\|<\cdot>^{a M-1} f\right\|,
$$

which shows

$$
\begin{aligned}
& \left\|x^{\gamma} G(t, s) f\right\| \leq\left\|G(t, s)\left(x^{\gamma} f\right)\right\|+C|t-s|\left\|<\cdot>^{a M-1} f\right\| \\
& \leq e^{K(t-s)^{2}}\left\|x^{\gamma} f\right\|+C|t-s|\left\|<\cdot>^{a M-1} f\right\|
\end{aligned}
$$

for $|\gamma|=a M$ from (1.14).

Let $\gamma$ be a multi-index such that $|\gamma|=a$. From (4.17) and (5.2) we write

$$
\partial_{x}^{\gamma}\left(G_{\epsilon}(t, s) f\right)=G_{\epsilon}(t, s)\left(\partial_{x}^{\gamma} f\right)+\sum_{\kappa<\gamma} P_{\kappa}(t, s) \partial_{x}^{\kappa} f
$$

where we have

$$
p_{\kappa}(t, s ; x, y)=w \cdot p_{\kappa}^{(1)}(t, s ; x y)+(t-s) p_{\kappa}^{(2)}(t, s ; x, y), w=y-x
$$

and

$$
\left|\partial_{x}^{\alpha} \partial_{y}^{\beta} p_{\kappa}^{(j)}(t, s ; x, y)\right| \leq C_{\alpha, \beta}\left(<x>^{M}+<y>^{M}\right)^{(a-|\kappa|)}, 0 \leq s, t \leq T
$$


for all $\alpha$ and $\beta$ and $j=1,2$ as in the proof of (4.19) and (4.20). Consequently as in the proof of Theorem 4.2 or (4.21) we get

$$
\left\|P_{\kappa}(t, s) \partial_{x}^{\kappa} f\right\| \leq C|t-s|\left\|<\cdot>^{M(a-|\kappa|)} \partial_{x}^{\kappa} f\right\|
$$

and so as in the proof of $(4.25)$

$$
\left\|P_{\kappa}(t, s) \partial_{x}^{\kappa} f\right\| \leq C^{\prime}|t-s|\|f\|_{B_{M}^{a}}
$$

which shows

$$
\begin{aligned}
& \left\|\partial_{x}^{\gamma}(G(t, s) f)\right\| \leq\left\|G(t, s)\left(\partial_{x}^{\gamma} f\right)\right\|+C^{\prime \prime}|t-s|\|f\|_{B_{M}^{a}} \\
& \leq e^{K(t-s)^{2}}\left\|\partial_{x}^{\gamma} f\right\|+C^{\prime \prime}|t-s|\|f\|_{B_{M}^{a}}
\end{aligned}
$$

from (1.14) and (5.7). Hence we obtain

$$
\|G(t, s) f\|_{B_{M}^{a}} \leq e^{K(t-s)^{2}}\|f\|_{B_{M}^{a}}+C_{a}|t-s|\|f\|_{B_{M}^{a}} \leq e^{K_{a}|t-s|}\|f\|_{B_{M}^{a}}
$$

together with (5.6), which completes the proof.

The theorem below has been proved in Example 1.1, p.329 of [15].

TheOREM 5.2. We assume (2.6) and (2.7). Let $0 \leq s \leq T$ and consider the Dirac equation (1.2) with $u(s)=f \in B_{M+1}^{a}(a=0,1,2, \ldots)$. Then there exists a unique solution $U(t, s) f \in \mathcal{E}_{t}^{0}\left([0, T] ; B_{M+1}^{a}\right) \cap \mathcal{E}_{t}^{1}\left([0, T] ; B_{M+1}^{a-1}\right)$, which satisfies

$$
\begin{aligned}
& \|U(t, s) f\|=\|f\|, \quad\|U(t, s) f\|_{B_{M+1}^{a}} \leq C_{a}(T)\|f\|_{B_{M+1}^{a}} \\
& (a=1,2, \ldots), \quad 0 \leq s, t \leq T .
\end{aligned}
$$

Proposition 5.3. Under the assumptions of Theorem 2.2 we have

$$
\|G(t, s) f-U(t, s) f\|_{B_{M+1}^{a}} \leq C_{a}(t-s)^{2}\|f\|_{B_{M+1}^{a+2}}, 0 \leq s, t \leq T
$$

for $a=0,1,2, \ldots$ and $f \in \mathcal{S}$. 
Proof. Let us write $\rho=t-s$ as in $\S 4$. From (4.1) we have

$$
\begin{aligned}
& i \frac{G_{\epsilon}(s+\rho, s) f-f}{\rho}=\int_{0}^{1} i \frac{\partial G_{\epsilon}}{\partial t}(s+\theta \rho, s) f d \theta \\
& =\int_{0}^{1}\left\{H(s+\theta \rho) G_{\epsilon}(s+\theta \rho, s) f+R_{\epsilon}(s+\theta \rho, s) f\right\} d \theta
\end{aligned}
$$

and so

$$
\begin{aligned}
& i \frac{G_{\epsilon}(s+\rho, s) f-f}{\rho}-H(s) f=\int_{0}^{1} R_{\epsilon}(s+\theta \rho, s) f d \theta+\int_{0}^{1} H(s+\theta \rho) \\
& \cdot\left\{G_{\epsilon}(s+\theta \rho, s) f-f\right\} d \theta+\int_{0}^{1}\{H(s+\theta \rho)-H(s)\} f d \theta .
\end{aligned}
$$

In the same way we have

$$
\begin{aligned}
& i \frac{U(s+\rho, s) f-f}{\rho}-H(s) f=\int_{0}^{1} H(s+\theta \rho)\{U(s+\theta \rho, s) f-f\} d \theta \\
& +\int_{0}^{1}\{H(s+\theta \rho)-H(s)\} f d \theta .
\end{aligned}
$$

Consequently we get

$$
\begin{aligned}
& i \frac{G_{\epsilon}(s+\rho, s) f-U(s+\rho, s) f}{\rho}=\int_{0}^{1} R_{\epsilon}(s+\theta \rho, s) f d \theta+\int_{0}^{1} H(s+\theta \rho) \\
& \cdot\left\{G_{\epsilon}(s+\theta \rho, s) f-f\right\} d \theta-\int_{0}^{1} H(s+\theta \rho)\{U(s+\theta \rho, s) f-f\} d \theta
\end{aligned}
$$

which shows

$$
\begin{aligned}
& i \frac{G(s+\rho, s) f-U(s+\rho, s) f}{\rho}=\int_{0}^{1} R(s+\theta \rho, s) f d \theta+\int_{0}^{1} H(s+\theta \rho) \\
& \cdot\{G(s+\theta \rho, s) f-f\} d \theta-\int_{0}^{1} H(s+\theta \rho)\{U(s+\theta \rho, s) f-f\} d \theta
\end{aligned}
$$

for $f \in \mathcal{S}$ from Proposition 3.2 and Theorem 4.2 as $\epsilon \rightarrow 0$. Applying Theorem 4.3 to (5.14), we get

$$
\begin{aligned}
& \frac{1}{|\rho|}\|G(t, s) f-U(t, s) f\|_{B_{M+1}^{a}} \leq C_{a}\left(|\rho|\|f\|_{B_{M+1}^{a+1}}+\int_{0}^{1} \| G(s+\theta \rho, s) f\right. \\
& \left.-f\left\|_{B_{M+1}^{a+1}} d \theta+\int_{0}^{1}\right\| U(s+\theta \rho, s) f-f \|_{B_{M+1}^{a+1}} d \theta\right) .
\end{aligned}
$$


Now we go back to (5.13). Then it follows from (4.15) and (4.26) that

$$
\begin{aligned}
& \frac{1}{|\rho|}\|G(s+\rho, s) f-f\|_{B_{M+1}^{a+1}} \leq C_{a} \int_{0}^{1}\left(\|G(s+\theta \rho, s) f\|_{B_{M+1}^{a+2}}\right. \\
& \left.+\|R(s+\theta \rho, s) f\|_{B_{M+1}^{a+1}}\right) d \theta \leq C_{a}^{\prime}\|f\|_{B_{M+1}^{a+2}} .
\end{aligned}
$$

In the same way it follows from (5.11) that

$$
\frac{1}{|\rho|}\|U(s+\rho, s) f-f\|_{B_{M+1}^{a+1}} \leq C_{a}^{\prime}\|f\|_{B_{M+1}^{a+2}} .
$$

Hence together with (5.15) we obtain

$$
\|G(t, s) f-U(t, s) f\|_{B_{M+1}^{a}} \leq C_{a} \rho^{2}\|f\|_{B_{M+1}^{a+2}}
$$

with another constant $C_{a}$, which shows (5.12).

Let us prove Theorem 2.1. We first state the lemma below, which has been proved in Lemma 6.1 of [17].

Lemma 5.4. Under the assumptions of Theorem 2.1 there exists an electromagnetic potential $(V, A)$ such that $V=0$ and

$$
\left|\partial_{x}^{\alpha} A_{j}(t, x)\right|+\left|\partial_{t} \partial_{x}^{\alpha} A_{j}(t, x)\right| \leq C_{\alpha}, \quad|\alpha| \geq 1
$$

in $[0, T] \times R^{d}$ for $j=1,2, \ldots, d$.

For a while we fix the electromagnetic potential $(V, A)$ found in Lemma 5.4. We note that this $(V, A)$ satisfies (2.6), (2.7) and (2.8) for all $\alpha$ with $M=1$. Applying Proposition 3.2 to (1.12), we have (1.16) for $f \in \mathcal{S}$. So we can see from Theorem 3.3 that $K_{D \Delta}(t, 0)$ can be extended to a bounded operator on $L^{2}$. 
Let $|\Delta| \leq 1$ and $f \in B_{2}^{2}$. From (1.16) we have

$$
\begin{aligned}
& K_{D \Delta}(t, 0) f-U(t, 0) f=G\left(t, \tau_{\nu-1}\right) \cdots G\left(\tau_{1}, 0\right) f-U\left(t, \tau_{\nu-1}\right) \cdots U\left(\tau_{1}, 0\right) f \\
& =\sum_{j=1}^{\nu} G\left(t, \tau_{\nu-1}\right) \cdots G\left(\tau_{j+1}, \tau_{j}\right)\left\{G\left(\tau_{j}, \tau_{j-1}\right)-U\left(\tau_{j}, \tau_{j-1}\right)\right\} U\left(\tau_{j-1}, 0\right) f .
\end{aligned}
$$

Applying (1.7), (1.14), (5.11) and (5.12) to (5.18), we get

$$
\begin{aligned}
& \left\|K_{D \Delta}(t, 0) f-U(t, 0) f\right\| \leq C \sum_{j=1}^{\nu} e^{K L_{0}}\left(\tau_{j}-\tau_{j-1}\right)^{2}\left\|U\left(\tau_{j-1}, 0\right) f\right\|_{B_{2}^{2}} \\
& \leq C^{\prime}|\Delta| L_{0} e^{K L_{0}}\|f\|_{B_{2}^{2}}
\end{aligned}
$$

with constants $C$ and $C^{\prime}$.

Let $f \in L^{2}$. For any $\epsilon>0$ we take a $g \in B_{2}^{2}$ such that $\|g-f\|<\epsilon$. Then from (1.14), (5.11) and (5.19) we have

$$
\begin{aligned}
& \left\|K_{D \Delta}(t, 0) f-U(t, 0) f\right\| \leq\left\|K_{D \Delta}(t, 0) g-U(t, 0) g\right\|+\left\|K_{D \Delta}(t, 0)(f-g)\right\| \\
& +\|U(t, 0)(f-g)\| \leq C^{\prime}|\Delta| L_{0} e^{K L_{0}}\|g\|_{B_{2}^{2}}+e^{K L_{0}}\|f-g\|+\|f-g\| \\
& \leq C^{\prime}|\Delta| L_{0} e^{K L_{0}}\|g\|_{B_{2}^{2}}+\left(1+e^{K L_{0}}\right) \epsilon .
\end{aligned}
$$

Hence we can prove that as $|\Delta| \rightarrow 0$ under the assumption $(1.7), K_{D \Delta}(t, 0) f$ converges to the solution $U(t, 0) f$ to $(1.2)$ with $u(0)=f$ in $L^{2}$ uniformly in $t \in[0, T]$.

Let $0<t_{1}<t$. We take arbitrary subdivisions $\Delta_{1}$ and $\Delta_{2}$ of $\left[0, t_{1}\right]$ and $\left[t_{1}, t\right]$ respectively. Let us consider the subdivision $\Delta=\Delta_{1} \cup \Delta_{2}$ of $[0, t]$. The point $t_{1}$ is included in $\Delta$ as a subdividing point. Then from (1.16) we have

$$
K_{D \Delta}(t, 0) f=K_{D \Delta_{2}}\left(t, t_{1}\right) K_{D \Delta_{1}}\left(t_{1}, 0\right) f
$$

for $f \in \mathcal{S}$ and so $f \in L^{2}$. So, letting $|\Delta| \rightarrow 0$, we can prove the first equation of the rule of two events (2.3) from the convergence of $K_{D \Delta}(t, 0) f$ to $K_{D}(t, 0) f$ 
proved above and the stability (1.14). In the same way we can also prove the second equation of (2.3).

Let us consider the gauge transformation (2.4). Let us write $S(t, s ; x, \xi, y)$ defined by $(1.10)$ for $\left(V^{\prime}, A^{\prime}\right)$ as $S^{\prime}(t, s ; x, \xi, y)$. Then we have

$$
S^{\prime}(t, s ; x, \xi, y)=S(t, s ; x, \xi, y)+\psi(t, x)-\psi(s, y)
$$

Since $\left(G_{\epsilon}^{\prime}(t, s) f\right)(x)=e^{i \psi(t, x)} G_{\epsilon}(t, s)\left(e^{-i \psi(s, \cdot)} f\right)(x)$ holds from $(1.11), K_{D \Delta}^{\prime}(t, 0) f=$ $e^{i \psi(t, \cdot)} K_{D \Delta}(t, 0)\left(e^{-i \psi(0, \cdot)} f\right)$ follows from (1.16). Hence we have (2.5) for $f \in L^{2}$. Thus we could complete the proof of Theorem 2.1 for $(V, A)$ found in Lemma 5.4 .

Let $\left(V^{\prime}, A^{\prime}\right)$ be a general electromagnetic potential for $E$ and $(B)_{1 \leq j<k \leq d}$ in Theorem 2.1 such that $V^{\prime}, \partial_{x_{j}} V^{\prime}, \partial_{t} A_{k}^{\prime}$ and $\partial_{x_{j}} A_{k}^{\prime}(j, k=1,2, \ldots, d)$ are continuous in $[0, T] \times R^{d}$. Let $(V, A)$ be the potential found in Lemma 5.4. Then, as was proved in p. 1024 of [17], there exists a real-valued function $\psi(t, x)$ such that $\partial_{x_{j}} \partial_{x_{k}} \psi$ and $\partial_{t} \partial_{x_{k}} \psi$ are continuous in $[0, T] \times R^{d}$ and (2.4) holds. We know in the above that (2.5) holds. We proved the results (1)-(5) of Theorem 2.1 for $(V, A)$. Thus we can easily complete the proof of Theorem 2.1 for $\left(V^{\prime}, A^{\prime}\right)$ from $(2.5)$.

As in the proof of Theorem 2.1 we can prove Theorem 2.2 from Theorems 5.1, 5.2 and Proposition 5.3.

Remark 5.1. In the present paper, to prove Theorems 2.1 and 2.2 we used the existence theorem of the solutions to the Dirac equation (1.2) stated in Theorem 5.2. This idea is originated in [24]. Here we note that following the proof of Theorem 1 in [19], we can prove directly Theorems 2.1 and 2.2 in the present paper from Theorems 3.3, 4.3 and 5.1 without the existence theorem, i.e. Theorem 5.2 to (1.2), though the proof is more complicated than ours. 


\section{References}

[1] Blanchard, Ph., Combe, Ph., Sirugue, M., Sirugue-Collin, M.: Jump processes related to the two dimensional Dirac equation. In: Stochastic Processes - Mathematics and Physics II (Bielefeld, 1985), Lecture Notes in Math. 1250, Berlin-Heidelberg: Springer-Verlag, 1987, pp. 1-13

[2] Calderón, A.P., Vaillancourt, R.: On the boundedness of pseudodifferential operators. J. Math. Soc. Japan 23, 374-378 (1971)

[3] Cartier, P., DeWitt, C.M.: Functional Integration: Action and Symmetries. Cambridge: Cambridge University Press, 2006

[4] Dirac, P.A.M.: The Principles of Quantum Mechanics, 4th edition. Oxford: Oxford University Press, 1958

[5] Dyson, F.: Comment on the topic "Beyond the black hole". In: Some Strangeness in the Proportion: A Centennial Symposium to Celebrate the Achievements of Albert Einstein. Reading, MA: Addison-Wesely, 1980, pp. $376-380$

[6] Feynman, R.P.: Theory of positrons. Phys. Rev. 76, 749-759 (1949)

[7] Feynman, R.P.: Space-time approach to quantum electrodynamics. Phys. Rev. 76, 769-789 (1949)

[8] Feynman, R.P.: Mathematical formulation of the quantum theory of electromagnetic interaction. Phys. Rev. 80, 440-457 (1950)

[9] Feynman, R.P., Hibbs, A.R.: Quantum Mechanics and Path Integrals. New York: McGraw-Hill, 1965 
[10] Feynman, R.P.: QED: The Strange Theory of Light and Matter. Princeton, NJ: Princeton University Press, 1985

[11] Folland, G.B.: Quantum Field Theory: A Tourist Guide for Mathematicians. Providence, Rhode Island: American Mathematical Society, 2008

[12] Gaveau, B.: Representation formulas of the Cauchy problem for hyperbolic systems generalizing Dirac system. J. Funct. Anal. 58, 310-319 (1984)

[13] Ichinose, T.: Path integral for the Dirac equation in two space-time dimensions. Proc. Japan Acad. 58 A, 290-293 (1982)

[14] Ichinose, T.: Path integral for the Dirac equation [translated of Sûgaku 42 (1990), 219-230]. Sugaku Exposition 6, 15-31 (1993)

[15] Ichinose, W.: A note on the existence and $\hbar$-dependency of the solution of equations in quantum mechanics. Osaka J. Math. 32, 327-345 (1995)

[16] Ichinose, W.: On the formulation of the Feynman path integral through broken line paths. Commun. Math. Phys. 189, 17-33 (1997)

[17] Ichinose, W.: On convergence of the Feynman path integral formulated through broken line paths. Rev. Math. Phys. 11, 1001-1025 (1999)

[18] Ichinose, W.: The phase space Feynman path integral with gauge invariance and its convergence. Rev. Math. Phys. 12, 1451-1463 (2000)

[19] Ichinose, W.: Convergence of the Feynman path integral in the weighted Sobolev spaces and the representation of correlation functions. J. Math. Soc. Japan 55, 957-983 (2003) 
[20] Ichinose, W.: A mathematical theory of the Feynman path integral for the generalized Pauli equations. J. Math. Soc. Japan 59, 649-668 (2007)

[21] Jacobson, T.: Spinor chain path integral for the Dirac equation. J. Phys. A: Math. Gen. 17, 2433-2451 (1984)

[22] Krauss, L.M.: Quantum Man: Richard Feynman's Life in Science. New York: W. W. Norton and Company, 2011

[23] Kumano-go, H.: Pseudo-Differential Operators. Cambridge, MA: MIT Press, 1981

[24] Lax, P.D., Richtmyer, R.D.: Survey of the stability of linear finite difference equations. Comm. Pure Appl. 9, 267-293 (1956)

[25] Peskin, M.E., Schroeder, D.V.: An Introduction to Quantum Field Theory. Cambridge, MA: Westview Press, 1995

[26] Rosen, G.: Formulations of Classical and Quantum Dynamical Theory. New York: Academic Press, 1969

[27] Swanson, M.S.: Path Integrals and Quantum Processes. San Diego: Academic Press, 1992 